\title{
Clinical, Histological and Direct Immunofluorescence Study in Immunobullous Diseases
}

\author{
Dr.S.Nageswaramma ${ }^{1}$, Dr.K.Hanuma Naik ${ }^{2}$, Dr.T.V.Narasimha Rao ${ }^{3}$ \\ 1,2,3 (Department of DVL, Guntur medical college, India )
}

\begin{abstract}
The autoimmune bullous diseases are characterised by pathogenic auto antibodies directed at target antigens whose function is either cell-cell adhesion within epidermis or adhesion of epidermis to dermis. The present study was under taken to study the incidence of immunobullous disorders and their clinical, histological and immunological features. The study was conducted for one year, clinically diagnosed cases of immunobullous disorders of both sexes were included in the study. Tzanck smear, HPE and Direct immunofluorescence were done for all the cases. A total of 20 new cases were diagnosed and registered for the study. Of these bullous pemphigoid was most commonly seen clinically, followed by pemphigus vulgaris and pemphigus foliaceous. On HPE sub epidermal blisters are seen in $70 \%$.Direct immunofluorescence was positive in $85 \%$ of cases and negative in 15\%. Direct immunofluorescence is an essential diagnostic tool for iimunobullous disorders.
\end{abstract}

Keywords: autoimmune, Direct immunoflourescence, immunobullous, pemphigoid.

\section{Introduction}

The auto immune bullous diseases are characterised by pathogenic autoantibodies against components of desmosomes and hemidesmosomes resulting in fluid filled lesions which may involve any area of skin and mucous membrane ${ }^{[1]}$. The bullous diseases although uncommon have a dramatic impact on the patient and their family and have severe economic consequences for the family and health services. The diseases can be debilitating and possibly fatal. Though various bullous diseases can be clinically differentiated from one another by morphological characteristics of the bullae, their patterns and distribution of the lesions, involvement of mucous membranes, the most important techniques for the investigation of patients with immunobullous diseases are histopathology, direct and indirect immunofluoresence.

\section{Aim}

The present study was undertaken to study the incidence of autoimmune bullous disorders among the outpatients, to correlate clinical/ histopathological/ immunological findings and classify them using histopathological and immunological analysis.

\section{Materials and methods}

A total of 20 cases of both sexes were clinically diagnosed as autoimmune bullous disorders. All patients were subjected to detailed history taking and clinical examination. Their particulars regarding age, sex, occupation, personal and family history presenting complaints, duration, general condition and findings on clinical examination were recorded in the proforma. Routine haematological and biochemical investigations were done and reports recorded. Tzanck smear was done at bedside, skin biopsy specimens for histopathological study were collected in formalin and for immunological study the specimens were collected in michel's medium. Their histological features, immunological features were studied and noted.

\section{Results}

Out of 20,000 new cases attending OPD 20 cases of immunobullous diseases were diagnosed clinically giving an incidence of $1 \%$.. Of these 20 cases $9(45 \%)$ were males and $11(55 \%)$ were female. Maximum number of patients were in the age group of 51 to 60 years which constitute 30\% ( TABLE 1)

Table 1

\begin{tabular}{|l|l|l|l|}
\hline SL.NO & AGE GROUP IN YRS & NO. & PERCENTAGE \\
\hline 1. & $1-10$ & 1 & $5 \%$ \\
\hline 2. & $11-20$ & 2 & $10 \%$ \\
\hline 3. & $21-30$ & 4 & $20 \%$ \\
\hline 4. & $31-40$ & 1 & $5 \%$ \\
\hline 5. & $41-50$ & 4 & $20 \%$ \\
\hline 6. & $51-60$ & 6 & $30 \%$ \\
\hline 7. & $61-70$ & 2 & $10 \%$ \\
\hline & TOTAL & 20 & $100 \%$ \\
\hline
\end{tabular}


Of these 20 cases 11 cases of bollous pemphigoid were diagnosed clinically contributing $55 \%$ in this study, 5 cases $(25 \%)$ of pemphigus vulgaris, pemphigus foliaceous, linear IgA dermatoses, dermatitis herpetiformis and bullous SLE constitute 1 case (5\%) each. (TABLE 2)

Table 2

\begin{tabular}{|l|l|l|l|}
\hline SL NO. & TYPE & NO. & PERCENTAGE \\
\hline 1. & BULLOUS PEMPHIGOID & 11 & $55 \%$ \\
\hline 2. & PEMPHIGUS VULGARIS & 5 & $25 \%$ \\
\hline 3. & PEMPHIGUS FOLIACEUS & 1 & $5 \%$ \\
\hline 4. & LINEAR IG A DERMATOSIS & 1 & $5 \%$ \\
\hline 5. & DERMATITIS HERPETIFORMIS & 1 & $5 \%$ \\
\hline 6. & BULLOUS SLE & 1 & $5 \%$ \\
\hline & TOTAL & 20 & $100 \%$ \\
\hline
\end{tabular}

11 patients(45\%) showed mucosal involvement. Of these 4 are pemphigus vulgaris and 5 are bullous pemphigoid. Of the 5 cases of pemphigus vulgaris 4 cases showed nikolsky sign positivity. Tzanck smear showed acantholytic cells i.e positive in 4 cases (20\%) and negative in $16(80 \%)$ cases. (TABLE 3)

Table 3

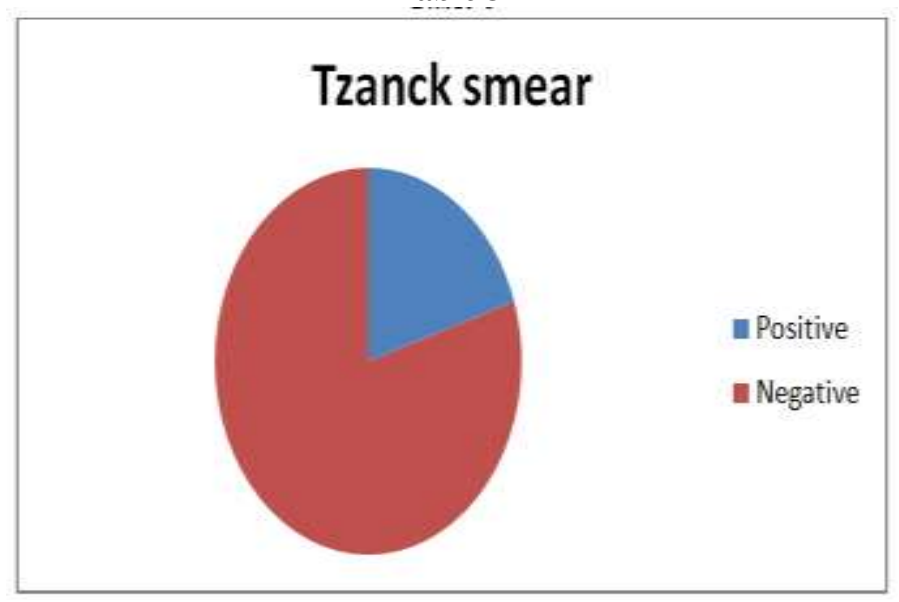

Histopathology showed subepidermal blister in 14 cases (70\%) and intra epidermal blister in 6 cases $(30 \%)$. Direct immunoflourescence is positive in 17 cases( $85 \%)$ and negative in 3 cases (15\%).

Out of 11 cases of bullous pemphigoid 8 cases showed positivity for Ig G and c3.

\section{Discussion}

In present study out of 20 cases of autoimmune bullous diseases diagnosed, bullous pemphigoid account for $55 \%$ followed by pemphigus vulgaris $25 \%$ which coincide with studies conducted by SM Lagan etal. ${ }^{[2]}$ According to study conducted by Kumar $\mathrm{KA}^{[3]}$ female preponderance in pemphigus vulgaris was noted. Female preponderance in pemphigus vulgaris is noted in present study. In bullous pemphigoid there is equal incidence in both the sexes (Stanley JR) ${ }^{[4]}$. In the present study there is higher incidence of bullous pemphigoid in male patients.

Bullous pemphigoid mainly occurs in the elderly, typically between 60 and 80 years of age. In the present study 6 out of 11 patients with bullous pemphigoid were in age group of 51-60 years constituting $54.4 \%$. Pemphigus is a disease of middle age, in the present study 3 out of 6 patients are in age group 41-50 years. According to Lopez-Robles E etal ${ }^{[5]}$, almost all pemphigus vulgaris have oral involvement. In present study 5 patients diagnosed as pemphigus vulgaris, $80 \%$ has oral involvement. Mucous membrane involvement in bullous pemphigoid ranges from $10-40 \%$, in the present study $45 \%$ of bullous pemphigoid have mucosal involvement.

Nikolsky 's sign is present in 4 out of $5(80 \%)$ patients of pemphigus vulgaris. Tzanck smear was also positive in 4 out of $5(80 \%)$ cases of pemphigus vulgaris. Out of the 14 subepidermal bullous disorders 11 cases (78.57\%) were bllous pemphigoid. So bullous pemphigoid is most common subepidermal bullous disorders which coincides with studies of National skin center and Bernard P etal ${ }^{[6]}$. Out of 20 patients in whom DIF study was carried out, it was found to be positive in $85 \%$ of the cases. In the present study pemphigus vulgaris patients with positive DIF showed intercellular IgG $80 \%, \mathrm{C} 3$ in $60 \%$. Bullous pemphigoid patients with positive DIF showed c3 in $90 \%$ cases, $\mathrm{IgG}$ in $81.8 \%, \mathrm{IgA}+\mathrm{C} 3$ 


\section{Conclusion}

The histological diagnosis of the various cutaneous autoimmune blistering diseases should always be confirmed with direct immunoflourescence studies. DIF testing is the prime diagnostic tool. DIF is a very reliable and sensitive diagnostic test.

\section{References}

[1]. Ahmed AR etal, pemphigus : current concepts, Ann intern-Med92(3),1980, 396-405

[2]. SM Langan, L Smeeth, R Hubbard, KM Flemming, C J P Smith and J West, Bullous pemphigoid and pemphigus vulgarisincidence and mortality in UK, BMJ 9-july, 2008, 337.

[3]. Kumar KA, incidence of pemphigus in thrissur district,south India, Indian J Dermatol venerol leprol, 74, 2008, 349-51.

[4]. Stanley JR: bullous pemphigoid, In: Freedberg IM, Eisen AZ,Wolff K etal, Fitzpatrick's Dermatology in general medicine, $5^{\text {th }}$ ed, ( New York: MC Grow Hill, 1999) 666-79.

[5]. Lopez-Robles E, Avalos-Diaz E, V ega-Memije E etal, TNF a, IL 6 are medicators in the blistering process of pemphigus, int J Dermatol 40, 2001, 188-9.

[6]. Bernard P, Valliant L, Labeille B,Bedane C, Arbeille B, Deneoux JP etal, Incidence and distribution of sub epidermal autoimmune bullous skin diseases in three French regions, Arch Dermatol 131(1), 1995, 48-52. 